\title{
COMPARAISON DE RUISSEAUX PERMANENTS ET TEMPORAIRES DE LA PROVENCE CALCAIRE
}

\author{
par P. Legier ${ }^{1}$ et J. TALiN ${ }^{1}$.
}

La comparaison de ruisseaux temporaires et permanents a été réalisée afin de déterminer leurs affinités. On a noté une stabilité des facteurs physiques et chimiques dans les ruisseaux permanents et pendant la phase lotique des ruisseaux temporaires; la phase stagnante des ruisseaux temporaires se révèle très instable. La comparaison des facteurs biotiques révèle l'affinité des ruisseaux permanents calmes et des ruisseaux temporaires. Les ruisseaux permanents à cours rapide et les ruisseaux temporaires sont peu affines. Quelques populations spécifiques peuvent survivre à la période sèche des ruisseaux temporaires, seulement lorsque la nappe phréatique est présente en profondeur.

\section{Comparison of intermittent and permanent streams of calcareous Provence.}

Comparison of intermittent and permanent streams has been made to determine their affinities. Stability of physical and chemical factors was observed in the permanent streams and in the lotic period of intermittent streams. The stagnant period of intermittent streams is unstable. The biotic comparisons have shown the affinities between permanent lentic streams and intermittent streams. Permanent lotic streams and intermittent streams show very little similarities. Some specific populations can maintain themselves during the dry period only when the phreatic sheet is present in depth.

\section{INTRODUCTION}

Le régime des précipitations et la nature karstique du sous-sol déterminent l'alternance d'une période d'inondation et d'une période d'assèchement pour bon nombre de ruisseaux méditerranéens. Parmi ce type de ruisseaux, il est possible de distinguer :

- les ruisseaux temporaires, caractérisés par une période d'inondation de plusieurs mois permettant l'installation des principaux groupes d'insectes aquatiques;

- les ruisseaux éphémères dont la période en cau, très brève,

1. Université d'Aix - MarseilleIII - Centre de Saint-Jérôme, Laboratoire de Biologie Animale-Ecologie, 13013 Marseille. 
permet l'installation d'une communauté réduite à quelques espèces de Diptères.

Dans l'état actuel de nos connaissances, cette distinction n'est encore qu'arbitraire mais nous l'accepterons comme hypothèse de travail. Nous serons amenés, par la suite, à préciser ces deux notions de «temporaire » et «d'éphémère ».

Les investigations, au demeurant peu nombreuses, dans le domaine des ruisseaux temporaires précédemment définis, sont essentiellement centrées sur la biologie de certaines espèces face à l'assèchement de leur biotope (Hynes, 1941; Hinton, 1953; Abell, 1959; Aubert, 1963; Khoo, 1964, 1968; Harrisson, 1966; Lehmkuhl, 1971). Peu d'auteurs se sont réellement intéressés à ce problème dans son ensemble, c'est-à-dire au niveau de l'écosystème; les premières recherches furent faites sur le continent américain par Stehr et Branson (1938), en Angleterre par Moon (1956), au Pays de Galles par Hynes (1958) et en Europe par Kamler et Riedel (1960). Clifford (1966) avait entrepris une étude écologique préliminaire des Invertébrés d'un ruisseau temporaire des Etats-Unis et Harrisson (1966) avançait certaines hypothèses au sujet du repeuplement d'un ruisseau temporaire en Rhodésie après son assèchement mais aucun de ces deux auteurs n'ont donné suite à leurs premières observations.

Dans le Sud de la France, les populations aquatiques affrontent un problème écologique de grande importance en rapport avec la sécheresse estivale. Les effets de la xéricité sont actuellement bien étudiés sur les biocénoses végétales et, si on connaît quelques travaux portant sur le peuplement de certains milieux terrestres (L. Bigo!, 1972) et aquatiques stagnants (A. Champeau, 1971), jusqu'à présent, l'influence de ce phénomène n'avait pas été abordée pour les communautés d'eau courante.

Nous nous proposerons donc d'étudier la structure des communautés qui peuplent ce type particulier de ruisseau et l'écologie des principales espèces qui constituent ces communautés. Cette note constitue le préambule de cette recherche. Nous déterminerons d'une part les affinités entre des ruisseaux temporaires différant par leur mode de mise en eau et par leur situation géographique et, d'autre part, les affinités de chacun d'eux avec des ruisseaux permanents de référence.

\section{1. - LOCALISATION DES STATIONS}

1.1. Ruisseau des Roques Hautes (altitude de la station : $264 \mathrm{~m}$ ).

C'est un ruisseau permanent issu d'une source importante qui correspond à un exutoire d'infiltration du barrage de Bimont (N.E. 
d'Aix-en-Provence, versant sud du massif de la Sainte-Victoire). La partie étudiée, longue de $1 \mathrm{~km}$ environ, est peu profonde. Le lit est constitué essentiellement de graviers et de blocs. La vitesse du courant est rapide $(80$ à $100 \mathrm{~cm} / \mathrm{s})$. Les zones calmes sont rares et la végétation limitée aux mousses. Le débit varie très peu au cours de l'année.

1.2. Ruisseau de Subéroque (altitude de la station : 430 à $300 \mathrm{~m}$ ).

Ce ruisseau temporaire est situé sur le versant Est du plateau du Cengle (face Sud du massif de Sainte-Victoire); il est formé par les eaux de ruissellement du massif de Sainte-Victoire et par les eaux de drainage du plateau. La partic prospectée est longue de $2 \mathrm{~km}$ environ. Faisant suite à une source intermittente, son lit est constitué d'une succession de cuves assez profondes $(40 \mathrm{~cm}$ à $1,5 \mathrm{~m}$ ), liées à la présence de barres rocheuses et de biotopes variés (sable, graviers, blocs). Le courant est en moyenne de 30 à $50 \mathrm{~cm} / \mathrm{s}$ en hiver. La végétation est rare : algues, mousses et quelques Characées dans les cuves. Pendant la période sèche, un suintement latéral alimente en permanence une seule cuve.

\subsection{Ruisseau de Péruy (altitude des stations : 440 et $295 \mathrm{~m}$ ).}

Il est situé près du massif de la Sainte-Baume, au-dessus de Saint-Zacharic. Une source captée alimente la partie permanente en amont de ce ruisseau. La vitesse du courant y est assez peu importante; on y trouve des biotopes à mousses, à hépatiques, des graviers fins et des galets. La partic aval $(6 \mathrm{~km})$ présente un lit assez large $(1,5$ à $2 \mathrm{~m})$, très rocailleux, avec des zones de roche nue. La période inondée est brève ( 4 mois environ); elle est caractérisée par des crues brutales et un courant rapide : 80 à $100 \mathrm{~cm} / \mathrm{s}$. Pendant la période sèche, seule une petite flaque étroite persiste.

\subsection{Le Destel (altitude des stations : $109 \mathrm{~m}$ ).}

Il coule dans une gorge étroite près d'Ollioules (Ouest de Toulon). C'est un ruisseau temporaire sur la plus grande partie de son cours. Nous avons choisi de prospecter une zone comprenant :

- en amont, une succession de cuves creusées dans des barres rocheuses; cette zone reste en permanence en eau, la vitesse du courant est nulle à l'exception des cascadelles qui unissent les cuves entre elles. La végétation est composée de mousses, algues filamenteuses, Characées et Juncacées. 
- en aval, des substrats variés; cette zone s'assèche pendant la saison estivale. Pendant la phase d'inondation, la vitesse du courant ne dépasse pas $40 \mathrm{~cm} / \mathrm{s}$.

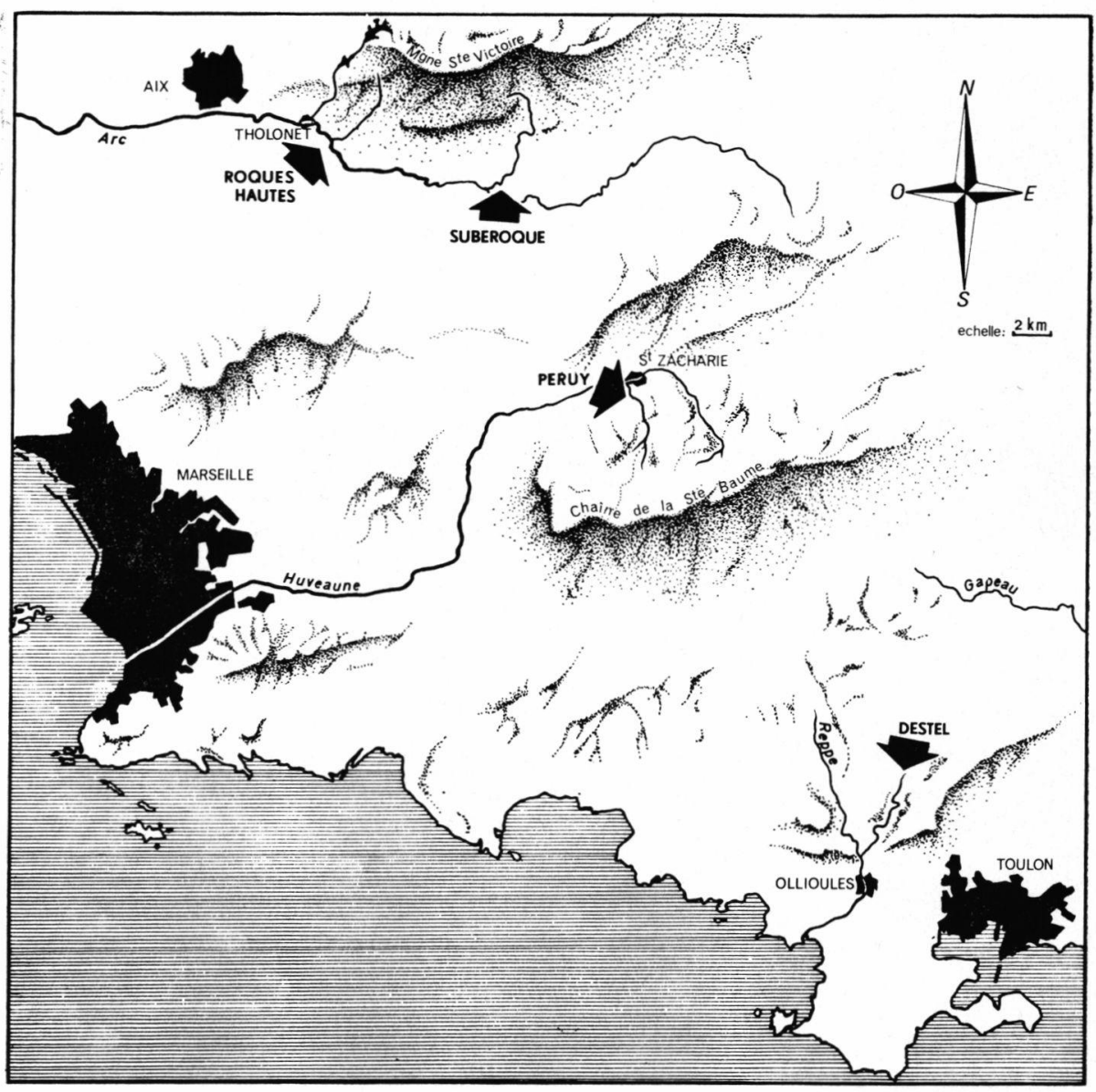

FIG. 1. - Localisation des ruisseaux prospectés.

\section{2. - PARAMETRES PHYSICO-CHIMIQUES}

Les composantes abiotiques de ces ruisseaux dépendent essentiellement de la durée de la période inondée. Dans les cours d'eau temporaires, on distingue trois phases obligatoires :

- la phase sèche (été et début de l'automne); 
- la phase en eau courante (hiver et début du printemps);

- la phase stagnante lorsque le courant devient trop faible pour assurer un renouvellement de l'eau jusqu'à l'assèchement total (printemps).

Ces phases sont de durées variables selon les ruisseaux. Elles sont étroitement liées aux précipitations atmosphériques et surtout à la profondeur de la nappe phréatique qui intervient pour une grande part dans la mise en eau.

\subsection{Matériel et méthodes.}

Nous avons effectué des relevés réguliers toutes les trois semaines dans chaque ruisseau (deux stations par ruisseau) :

- pour les prélèvements de la faune benthique, dans les forts courants, nous avons utilisé des filets de Surber dont la surface à échantillonner est de $225 \mathrm{~cm}^{2}$; le filet est maintenu immobile et le fond est râclé en amont de l'ouverture. En courant faible, la même opération est accompagnée de prélèvements réalisés à l'aide d'un filet à plancton de $10 \mathrm{~cm}$ de diamètre. La faune des galets est prélevée à la pince. La faune des mousses est récoltée par lavage et triée sur place. Le produit des récolles est ensuite conservé dans l'alcool à $70^{\circ}$.

- au cours de chacune des sorties effectuées, nous avons fait des mesures de température, d'oxygène dissous et de vitesse de courant. Nous avons également réalisé des prélèvements d'eau pour analyser les principaux sels dissous.

- afin de pouvoir suivre l'évolution des trois phases, rapide, calme et sèche des ruisseaux temporaires, nous avons cartographié dans chacun de ces ruisseaux une portion de cours, longue de $10 \mathrm{~m}$, comprenant plusieurs substrats; nous avons noté la surface inondée et calculé les pourcentages de recouvrement. Sur les figures 2 et 3 sont représentés, pour chaque station :

- les températures moyennes, minimales et maximales relevées au même moment de la journée entre zones à l'ombre et zones ensoleillées (janvier 1972 - mai 1973);

- la présence du courant (trait épais) (vitesse mesurée avec un tube de Pitot et un moulinet Ott);

- les relevés pluviométriques provenant des postes météorologiques régionaux les plus proches de nos stations (météorologie régionale) ;

- les pourcentages de surface inondée (grisé) (juin 1972-mai 1973). 

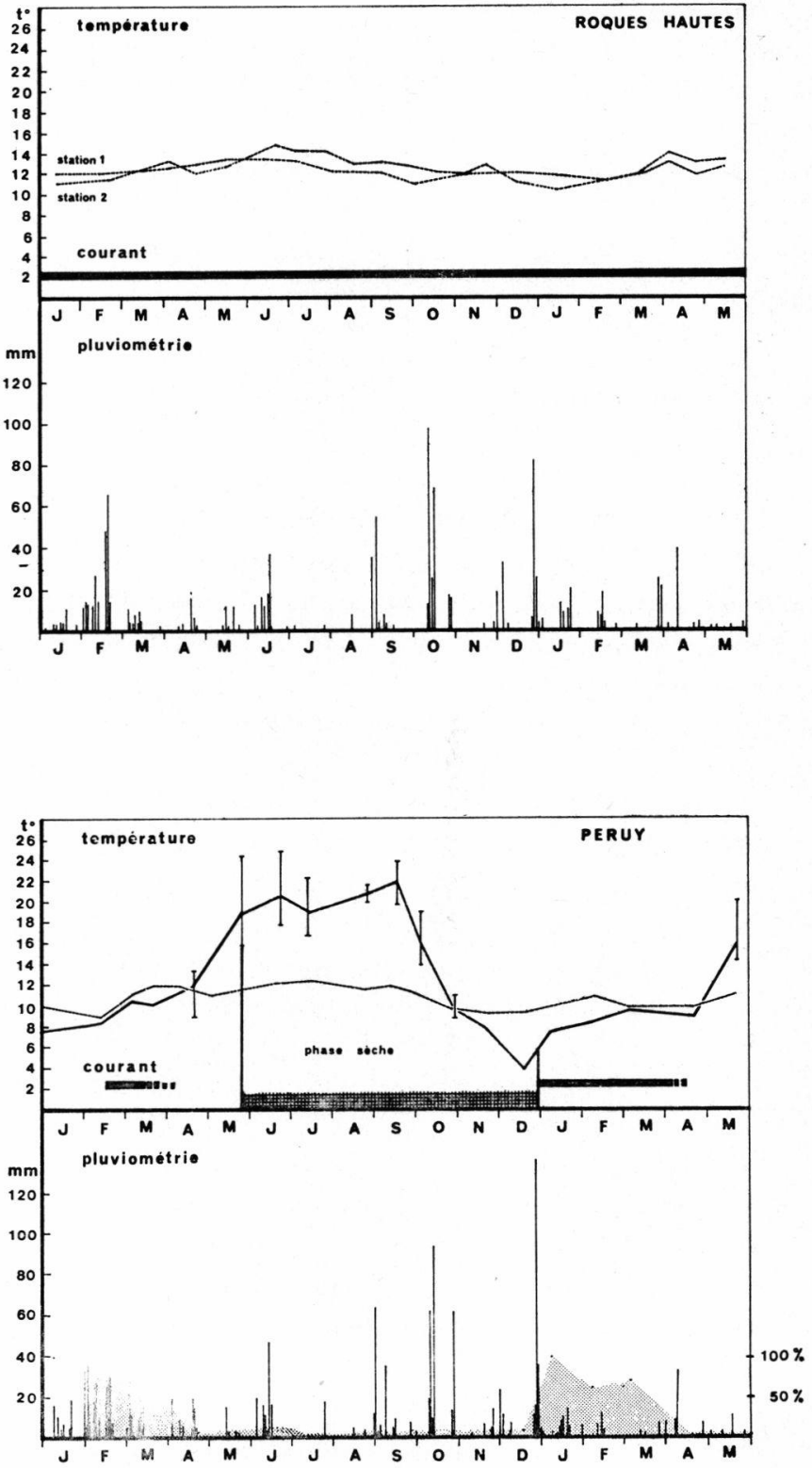

FIg. 2 et 3. - Facteurs abiotiques : Température de l'eau (trait plein : stations temporaires) - (trait pointillé : stations permanentes. - Pluviométrie et surfaces inondées (zones en grisé) - Présence du courant (trait plein épais : phase lotique) - (trait pointillé épais : phase stagnante). 

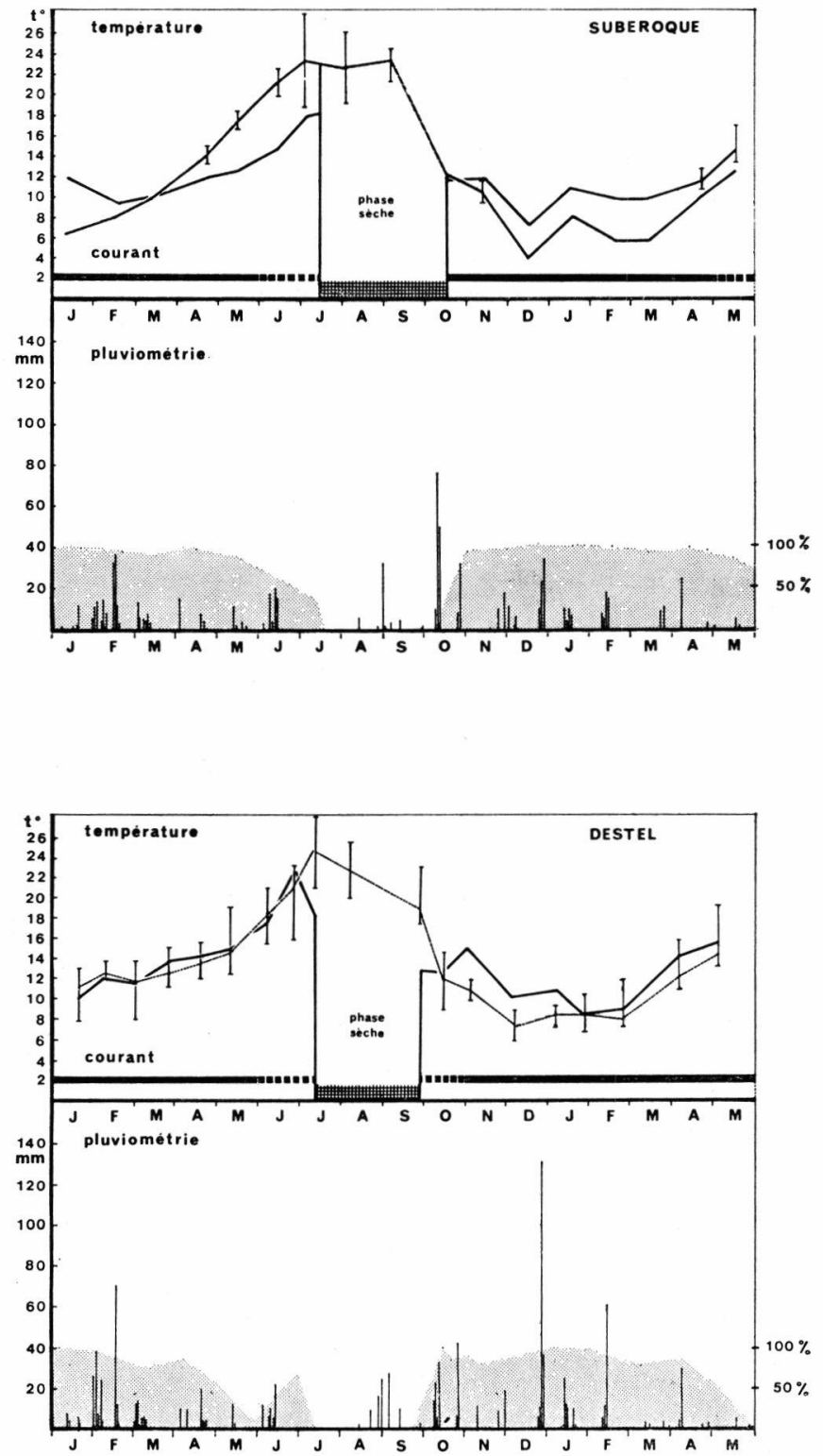


\subsection{Résultats.}

\subsubsection{LA TEMPÉRATURE.}

Les régimes thermiques des ruisseaux sont mis en évidence dans les figures 2 et 3 . Les relevés ont été effectués ponctuellement et à la même heure à chaque sortie; plusieurs mesures ont été faites, permettant ainsi de noter des températures maximales et minimales.

Les températures des ruisseaux permanents issus de sources (Roques Hautes : RH; station du ruisseau de Péruy : $\mathrm{PH}$ ) sont assez voisines et varient entre 9 et $13^{\circ} \mathrm{C}$ au cours de l'année (courbe en trait pointillé).

Dans les parties temporaires, les températures sont très variables et suivent les fluctuations climatiques saisonnières. Dès que le courant devient faible, on remarque une amplitude thermique de 3 à $10^{\circ} \mathrm{C}$ selon l'ensoleillement car la phase stagnante présente des flaques isolées, souvent peu profondes, où la température atteint parfois $30^{\circ} \mathrm{C}$ (courbe en trait plein).

\subsubsection{OXYGÈNE DISSOUS.}

La saturation en oxygène dissous est toujours proche de $100 \%$ pendant la phase lotique et légèrement inférieure à $100 \%$ dans les sources (Roques Hautes et Péruy).

A la fin $d u$ printemps, alors que la vitesse du courant diminue, la température de l'eau s'élève et entraîne une augmentation de la microflore (diatomées, algues filamenteuses). On observe alors deux phénomènes :

- une sursaturation en oxygène dissous : jusqu'à $150 \%$, dans les flaques larges et peu profondes;

— un déficit poussé en oxygène dissous : teneur inférieure à $40 \%$ dans les cuves profondes ou dans des fissures étroites. Ceci est très net dans les cuves du Destel et dans les flaques de Péruy aval (cf. le graphique 4 où ont figurés les pourcentages minimaux et maximaux observés : zones en grisé).

\subsubsection{Calcium et Magnésium.}

Tous les ruisseaux étudiés sont situés en terrain calcaire ou dolomitique et les teneurs en Ca et $\mathrm{Mg}$ sont assez élevées. 
Dans les parties permanentes, les quantités varient peu au cours de l'année : 80 à $100 \mathrm{mg}$ pour Péruy $(\mathrm{PH}), 65$ à 90 pour les Roques Hautes $(\mathrm{RH})$. Dans les parties temporaires, les teneurs sont élevées et relativement constantes pendant la phase lotique : 120 à $150 \mathrm{mg} / \mathrm{l}$.

Pendant la phase stagnante, on remarque une baisse très nette à Subéroque : de $140 \mathrm{mg}$ à $40 \mathrm{mg} \mathrm{Ca} / 1$ et de 80 à $8 \mathrm{mg} \mathrm{Mg} / 1$ pour les mois de septembre et octobre; ceci semble correspondre aux précipitations qui remplissent les dépressions d'eau pure très peu minéralisées avant la remise en eau du ruisseau.

\subsubsection{Autres SEls dissous.}

Plusieurs analyses de nitrates, nitrites, phosphates, ont été réalisées. Ces sels sont le plus souvent à l'état de traces ou dépassent rarement $40 \mathrm{mg} / \mathrm{l}$.

Les chlorures sont toujours inférieurs à $25 \mathrm{mg} / \mathrm{l}$.

Les ions $\mathrm{CO}_{3} \mathrm{H}^{-}$sont au contraire très abondants mais leur teneur varie beaucoup d'un prélèvement à l'autre. Ces teneurs oscillent entre 50 et $200 \mathrm{mg} / 1$ pour le Destel et les Roques Hautes, entre 80 et $250 \mathrm{mg} / \mathrm{l}$ pour Subéroque. Dans le ruisseau de Péruy, on remarque une teneur très élevée en $\mathrm{CO}_{3} \mathrm{H}$ à la source : 180 à $260 \mathrm{mg} / \mathrm{l}$ et beaucoup plus faible dans la partie aval temporaire : 60 à $180 \mathrm{mg} / \mathrm{l}$.

\section{3. - ANALYSE COMPARATIVE DES PEUPLEMENTS}

Les prélèvements de faune ont été effectués régulièrement pendant deux ans à raison d'un toutes les trois semaines par station. Le matériel récolté a été déterminé au laboratoire et toutes les larves d'insectes ont fait l'objet de mesures (capsule céphalique) afin de mettre en évidence leur cycle biologique.

\subsection{Composition biotique.}

La comparaison des peuplements des ruisseaux a été réalisée entre les trois stations permanentes de références (Péruy amont: PH; Destel amont : DH; Roques Hautes : RH) et les trois stations temporaires (Péruy aval, Destel aval et Subéroque). Il apparaît que les ruisseaux temporaires étudiés sont en général aussi riches que les ruisseaux permanents témoins : 

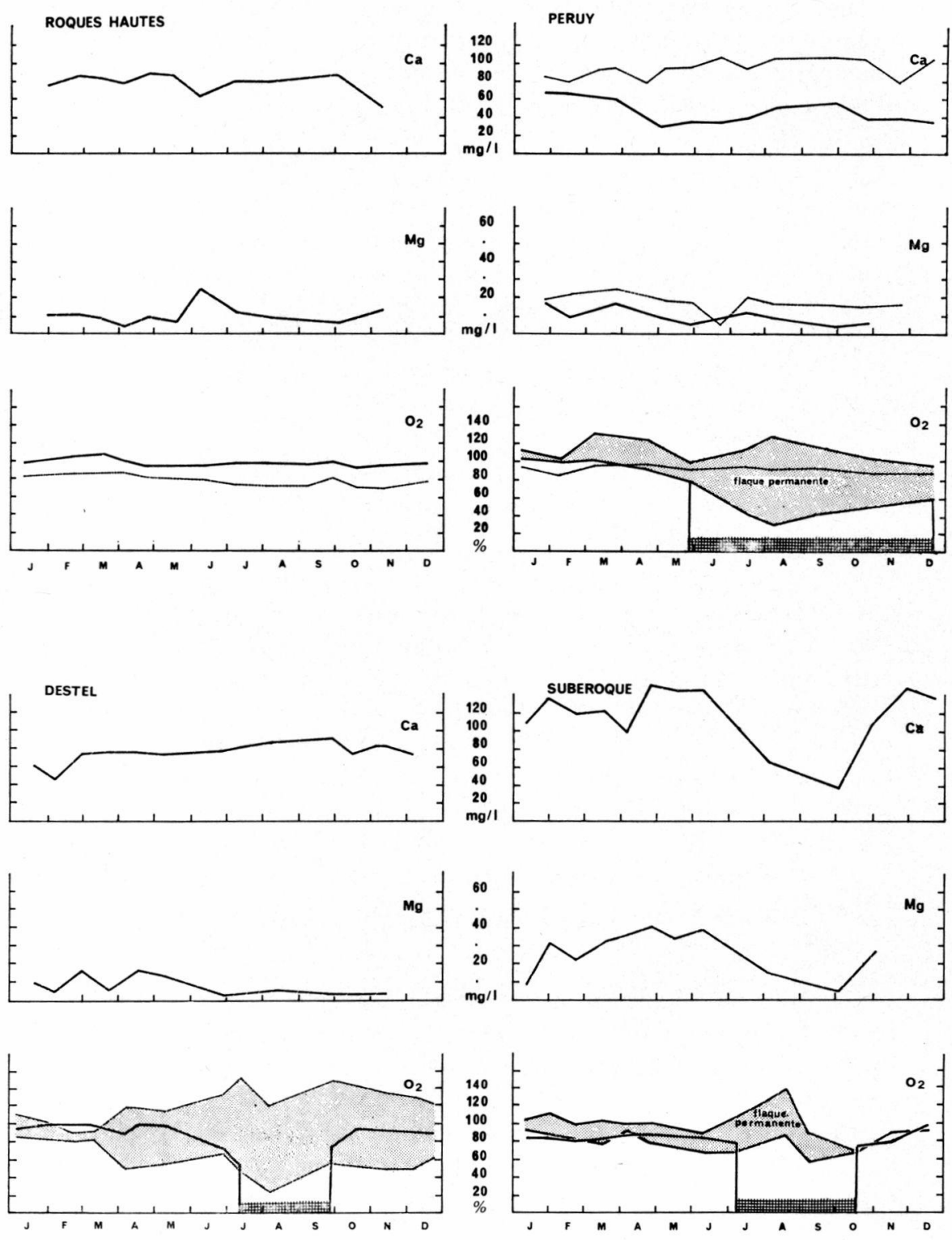

Fig. 4. - Facteurs abiotiques : pourcentages de saturation en $\mathrm{O}_{2}$ dissous et teneurs en ions $\mathrm{Ca}^{++}$et $\mathrm{Mg}^{++}$.

- $\mathrm{O}_{2}$ dissous : stations permanentes en trait pointillé; stations temporaires en trait plein. Les zones en grisé correspondent aux variations d'amplitude observées dans une même station.

- $\mathrm{Ca}^{++}$et $\mathrm{Mg}^{++}$: lorsque les teneurs sont semblables dans les deux stations, une seule courbe est figurée. Stations permanentes en trait pointillé; stations temporaires en trait plein. 
Ruisseaux permanents

Roques Hautes ..... 43 espèces

Destel amont ......6.64 espèces

Péruy amont $\ldots \ldots \ldots, 46$ espèces
Ruisseaux temporaires

Subéroque ....... 80 espèces

Destel aval .......6. 60 espèces

Péruy aval ...... 42 espèces

L'examen détaillé des inventaires de ces six stations révèle la prédominance des groupes limnophiles. Ainsi, quelle que soit la station, le groupe des Coléoptères Dytiscidés est le plus riche en espèces; les Hétéroptères sont représentés de façon constante dans les deux types de ruisseau. On note une faible participation des principaux groupes rhéophiles (Trichoptères Rhyacophilidés et Polycentropidés, Diptères Simuliidés et Éphéméroptères Baetidés).

\subsection{Les différences.}

\subsection{1. ÉlÉments de comparaison.}

Si on considère la proportion des espèces rhéophiles dans le peuplement des trois stations permanentes témoins, on obtient le classement suivant : Roques Hautes, Péruy amont, Destel amont.

Ainsi, pour le groupe des Trichoptères qui renferme à la fois des espèces limnophiles et des espèces rhéophiles, on constate que :

- Roques Hautes hébergent 6 espèces rhéophiles : Rhyacophila dorsalis, Hydropsyche instabilis, Synagapetus dubilans, Plectrocnemia conspersa, Silo nigricornis, Hydroptila sp. eł 3 espèces limnophiles : Micropterna testacea, Limnephilus griseus, Odontocerum albicorne.

- Péruy amont héberge 5 espèces rhéophiles : Hyporhyacophila sp., Rhyacophila vulgaris, Plectrocnemia conspersa, Silo nigricornis, Wormaldia occipitalis et 4 espèces limnophiles : Odontocerum albicorne, Micropterna testacea, Allogamus auricollis, Limnephilus griseus.

- Destel amont héberge 3 espèces rhéophiles : Plectrocnemia conspersa, Wormaldia occipitalis, Hydroptila sp., 3 espèces limnophiles : Micropterna testacea, Mystacides azurea, Limnephilus griseus.

Ce classement, établi selon le degré de rhéophilie du peuplement, est en accord avec la vitesse moyenne du courant de ces trois stations :

- Roques Hautes : vitesse du courant supérieure à $80 \mathrm{~cm} / \mathrm{s}$.

- Péruy amont : vitesse du courant comprise entre 40 et $80 \mathrm{~cm} / \mathrm{s}$.

- Destel amont : vitesse du courant inférieure à $20 \mathrm{~cm} / \mathrm{s}$. 
N. B. - Pour chacun de ces trois ruisseaux permanents, les vitesses du courant sont pratiquement identiques sur toute la longueur de la portion étudiée.

Nous considèrerons que « Roques Hautes» représente la station à cours rapide de référence et "Destel amont» la station calme témoin. Enfin, «Péruy amont» sera notre station intermédiaire.

Dans la comparaison des stations permanentes et des stations temporaires, nous avons choisi de distinguer pour les ruisseaux temporaires deux phases pendant la période inondée :

- une phase lotique pendant laquelle la mise en eau du cours est totale et la vitesse du courant maximale pour le ruisseau considéré;

- une phase stagnante à partir du moment où le courant devient nul et où les différentes dépressions du ruisseau sont isolées les unes des autres.

Ainsi, les trois stations temporaires étudiées possèdent des périodes inondées de durées différentes : deux ruisseaux sont en eau pendant 9 mois par an (station aval du ruisseau du Destel, ruisseau de Subéroque), la station aval du ruisseau de Péruy est en eau pendant 5 mois mais conserve en permanence une flaque de $0,50 \mathrm{~m}^{2}$. Les deux phases de cette période inondée sont de durées inégales au sein d'un même ruisseau et pour des ruisseaux différents : la phase lotique s'étend du mois d'octobre au mois d'avril pour les ruisseaux du Destel et de Subéroque, de la fin du mois de décembre jusqu'au mois d'avril pour le ruisseau de Péruy. Au niveau de la phase stagnante, les différences de durée sont moindres; elles sont de deux mois environ pour les ruisseaux du Destel et de Subéroque (mai, juin), d'un mois (mai) pour le ruisseau de Péruy (mais le peuplement de la flaque permanente persiste jusqu'en septembre).

Les peuplements de ces phases seront comparés entre eux et avec ceux des trois stations témoins. Nous avons donc établi des coefficients d'affinité cénotique entre 9 stations (voir tableau $n^{\circ} 5$, zone en grisé).

Les valeurs inscrites dans le tableau des contingences sont obtenues de Ia façon suivante :

- le nombre d'espèces communes aux deux biotopes considérés No figure sur la première ligne.

- le nombre théorique d'espèces communes Ne figure sur la deuxième ligne. Afin d'éliminer les espèces qui nous paraissent accidentelles, nous avons choisi de rejeter celles dont la fréquence dans les relevés était inférieure à $15 \%$. La valeur 
calculée du nombre d'espèces communes s'obtient à partir du nombre total d'espèces $\mathrm{Nt}$ présentes dans plus de $15 \%$ des prélèvements effectués dans chacun des biotopes (permanents, temporaires lotiques et temporaires stagnants). Si le biotope 1 renferme $\mathrm{n} 1$ espèces et le biotope $2 \mathrm{n} 2$ espèces, la valeur de $\mathrm{Nc}$ sera donnée par la relation :

$$
\mathrm{Nc}=\frac{\mathrm{n} 1 \times \mathrm{n} 2}{\mathrm{Nt}}
$$

La comparaison de la valeur théorique $\mathrm{Nc}$ au nombre observé d'espèces communes aux deux biotopes considérés No donne le sens de l'affinité (Dagnelic, 1960 in Cancela da Fonseca, 1966) :

No $>\mathrm{Ne}$ : l'affinité sera positive

No $=\mathrm{Nc}:$ l'affinité sera nulle

No $<\mathrm{Nc}$ : l'affinité sera négative.

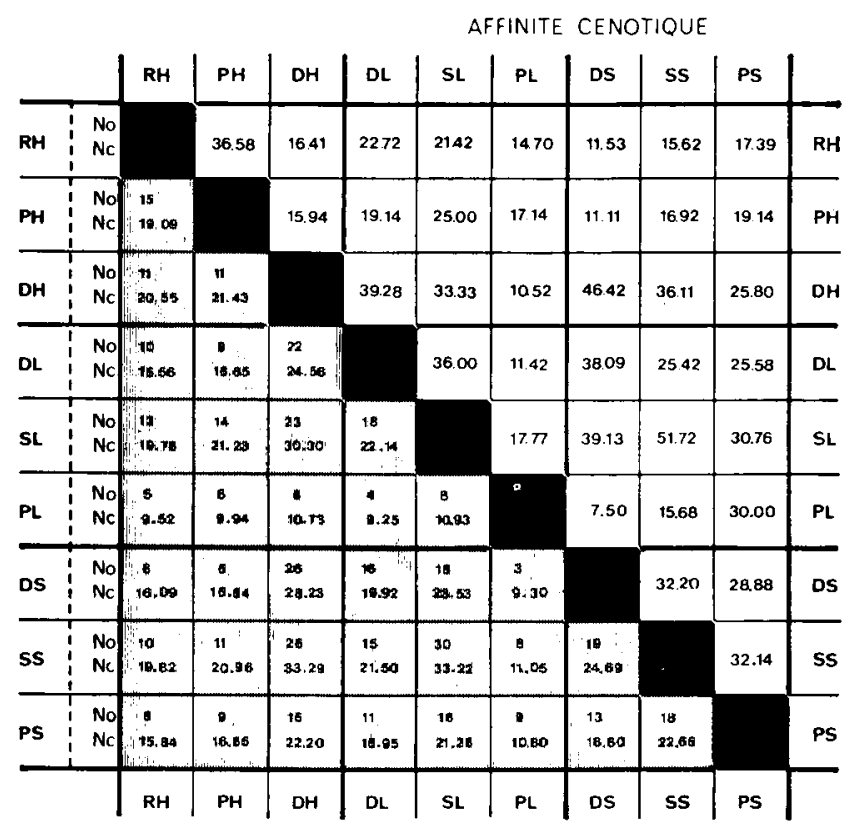

TABLEAU DE CONTINGENCE

FIg. 5. - Tableau des contingences et des affinités cénotiques : - partie grisée : calcul des contingences. No : nombre d'espèces communes à 2 stations; Ne : nombre théorique d'espèces communes à 2 stations. - partie claire : coefficients d'affinité de Jaccard. 


\subsubsection{Discussion DES DONNÉES DE COMPARAISON.}

\subsubsection{A perçu général.}

Pour chaque comparaison effectuée, on note que Nc est supérieur à No : les affinités sont donc négatives, ce qui montre a priori que les biotopes se ressemblent peu. Les différences observées entre No et Nc, testées par la méthode $\mathrm{du} \chi^{2}$ avec correction de Yates, sont pour la plupart hautement significatives.

Dans le cas de la comparaison entre les phases lotiques et stagnantes des portions temporaires d'un même ruisseau (Subéroque : SL/SS et Destel : DL/DS), la différence est significative. Par contre, dans le cas de la comparaison des deux phases de la partie temporaire du ruisseau de Péruy (PL/PS), la différence n'est pas significative. Dans ce dernier cas, la coexistence de certaines espèces dans les deux phases n'est probablement pas due uniquement au hasard mais à l'intervention d'un facteur que nous ne sommes pas encore en mesure de déterminer.

Bien que les affinités soient négatives, on note cependant une gradation dans ces "non corrélations» comme le montrent les valeurs du coefficient d'affinité cénotique de Jaccard (partie claire $\mathrm{du}$ tableau $\mathrm{n}^{\circ}$ 5).

\subsubsection{Comparaison des ruisseaux permanents entre eux.}

L'analyse des affinités entre les 1rois ruisseaux permanents pris deux à deux confirme et complète l'observation faite dans le paragraphe 3.2.1 : la station permanente du ruisseau de Péruy $(\mathrm{PH})$, déjà classé en position intermédiaire entre les ruisseaux permanents à cours rapides $(\mathrm{RH})$ et à cours lent $(\mathrm{DH})$, se rapproche, compte tenu des affinités de la station à cours rapide de référence. En effet, le cofficient le plus élevé apparaît lors de la comparaison de cette station avec le ruisseau des Roques Hautes $(36,58 \%)$ alors que les affinités entre la station $\mathrm{PH}$ ou le ruisseau RH et la partie permanente témoin du Destel (DH) sont inférieures à $17 \%$.

\subsubsection{Comparaison des ruisseaux permanents avec les ruis- seaux temporaires.}

- Au niveau de la phase lotique : on remarque que toutes les comparaisons d'affinités effectuées avec la phase lotique de la parti.e temporaire du ruisseau de Péruy (PL) présentent une coefficient très faible (inférieur à $18 \%$ ). Ceci semble lié, dans ce ruisseau, à la brièveté de la période inondée et en particulier à la brièveté de la phase lotique, qui ne permet pas l'installation d'un cortège faunistique important. 
Pour des stations présentant des périodes inondées plus longues et de même durée (SL, DL), leurs affinités avec les stations permanentes sont supérieures avec des coefficients compris entre 19 et $40 \%$.

Paradoxalement, pour ces ruisseaux temporaires à longue phase lotique, les affinités les plus élevées apparaissent lors des comparaisons avec le témoin permanent calme $\mathrm{DH}$; en effet, les affinités de ces phases lotiques temporaires avec ce témoin permanent $\mathrm{DH}$ se situent entre $33 \%$ et $40 \%$.

- Au niveau de la phase stagnante : les coefficients de Jacard sont faibles dans les comparaisons avec les témoins à cours rapide permanents $(\mathrm{RH}, \mathrm{PH})$; ils sont inférieurs à $20 \%$. Pour le témoin calme DH, les affinités sont plus élevées puisque les coefficients sont compris entre 25 et $47 \%$.

Ces résultats font apparaître l'importance de la durée de la mise en eau pour les ruisseaux temporaires : les stations où la période inondée est la plus longue sont celles dont le peuplement se raprapproche le plus de celui de la station permanente calme. Le facteur «durée de mise en eau » semble l'emporter sur la vitesse de I'écoulement.

\subsubsection{Comparaison des ruisseaux temporaires entre eux.}

- Au niveau des phases lotiques: lorsque les durées des périodes lotiques sont voisines, le coefficient est relativement élevé; il est de $36 \%$ dans le cas des comparaisons d'affinités réalisées entre les phases lotiques des ruisseaux temporaires du Destel et de Subéroque (DL/SL).

Les faunes lotiques hébergées dans les biotopes dont les durées de mise en eau sont différentes n'ont pas d'affinités entre elles : $11,42 \%$ pour DL/PL, $17,77 \%$ pour SL/PL.

- Au niveau des phases stagnantes : dans tous les cas, les valeurs du coefficient s'établissent autour de $30 \%$. Contrairement à leurs phases lotiques, les ruisseaux temporaires ont des phases stagnantes de durée à peu près égale. Ceci explique pour ces dernières leurs affinités voisines.

- Au niveau des phases lotiques et stagnantes : les coefficients sont plus élevés, ils oscillent entre 30 et $52 \%$ pour les deux phases d'un même ruisseau, entre 25 et $40 \%$ pour les deux phases comparées de ruisseaux différents. Les comparaisons d'affinités avec la phase lotique du ruisseau de Péruy donnent par contre des coefficients inférieurs à $16 \%$.

Ces résultats confirment une fois de plus l'importance de la durée de la mise en eau pour les ruisseaux temporaires : les affi- 
nités sont fortes lorsqu'on compare des phases de durée semblables (phases lotiques des ruisseaux de Subéroque et du Destel, phases stagnantes des ruisseaux de Péruy, Subéroque et Destel). Seules les comparaisons effectuées avec la phase lotique du ruisseau de Péruy fournissent des coefficients faibles; c'est au niveau de cette phase qu'on a remarqué des différences sensibles de durée d'inondation.

\subsection{Relations entre la composition biotique et les données de com- paraison.}

Les ruisseaux temporaires ont élé divisés en deux phases. La première, la phase lotique, correspond au début de la «Vie» du ruisseau. En effet, dès le mois d'octobre, apparaissent les premières larves dans les ruisseaux temporaires de Subéroque et du Destel et le développement de ces populations larvaires s'effectue jusqu'au mois d'avril; la durée du développement est donc de 7 mois. Par contre, les espèces du ruisseau temporaire de Péruy ne disposent que de 4 mois pour terminer leur évolution larvaire, d'où l'importance de la durée de cette phase. La deuxième phase, la phase stagnante, correspond à la fin de la «Vie» des ruisseaux temporaires. Elle est essentiellement caractérisée par la présence de nymphes et d'insectes aquatiques adultes. La durée de cette phase n'a donc d'importance qu'au niveau des nymphes, c'est-à-dire des individus qui n'ont pu terminer leur développement larvaire pendant la phase lotique. Ces populations nymphales doivent terminer leur évolution avant l'assèchement complet du biotope.

\subsubsection{IMPORTANCE DES DURÉES DES PHASES LOTIQUES.}

Le degré des affinités entre deux stations temporaires est directement influencé par la durée relative de leurs phases lotiques. Une phase lotique courte, comme celle de la station temporaire du ruisseau de Péruy, permet l'installation d'un peuplement composé essentiellement d'espèces à cycle court, surtout représenté par une espèce de Trichoptères (Micropterna testacea dont le cycle s'étale entre le mois d'octobre et le mois d'avril) et quatre groupes de Diptères (Chironomides, Culicidés, Cératopogonidés et Simuliidés : Simulium bezii).

Lorsque les durées des phases lotiques des ruisseaux temporaires sont plus longues (ruisseau de Subéroque et station aval du Destel), le cortège faunistique est plus important. Il se compose, en plus des espèces recensées au cours de la phase lotique du ruisseau de Péruy, d'espèces dont le développement préimaginal est plus 
long. Ces espèces appartiennent essentiellement à quatre groupes : groupes des Éphéméroptères (Habrophlebia fusca, H. lauta, Cloeon dipterum, Caenis macrura, Baetis sp.), des Plécoptères (Nemura cinerea pour le ruisseau de Subéroque, Isoperla grammatica pour les ruisseaux de Subéroque et du Destel), des Trichoptères (Limnephilus griseus) et des Odonates (Pyrrhosoma nymphula, Aeschna mixa et Lestes viridis).

La durée de la phase lotique sélectionne les espèces et conditionne donc la qualité du peuplement des ruisseaux temporaires. Pour qu'une espèce puisse s'y développer, il est nécessaire que son cycle biologique coïncide avec la période de mise en eau du ruisseau qu'elle habite; il faut qu'elle parvienne à la nymphose avant ou au début de la phase stagnante.

C'est le cas, entre autres, pour Allogamus auricollis qui se trouve en abondance dans la partie permanente, en amont du ruisseau de Péruy. Ses larves dérivent vers la partie temporaire aval de ce ruisseau dans une faible proportion, au moment de la remise en eau. Cependant, leur cycle biologique, qui s'étale sur 8 mois dans la zone permanente, ne pourra pas s'achever dans la zone temporaire avant l'assèchement. Un autre exemple est celui de Isoperla grammatica qu'on rencontre dans des ruisseaux temporaires qui possèdent une phase lotique longue (Subéroque, Destel) et qui est absente de la partie temporaire du ruisseau de Péruy alors qu'elle peuple en abondance la partie permanente de ce même ruisseau.

A côté des espèces dont la durée de la vie préimaginale coïncide avec la durée de la phase lotique du ruisseau temporaire, les seules espèces qui subsistent sont celles qui résistent à la période sèche soit en s'enfonçant avec la nappe phréatique lorsque la texture du substrat le permet (ex. : le Mollusque Ancylus sp., la Planaire Dugesia tigrina, les Coléoptères Oulimnius rivularis, Graptodytes ignotus et le Crustacé Asellus sp.), soit en entrant en vie latente (diapause de la larve de Plécoptère, Capnia bifrons, des Crustacés planctoniques Ostracodes et Cyclopides ou quiescence des larves de Diptères Cératopogonides).

\subsubsection{LE PRoblème des AFFinités des RUISSEAux TEMPoraires AVEC LES RUISSEAUX PERMANENTS.}

A l'issue de cette étude, nous avons constaté la ressemblance des phases lotiques et stagnantes des ruisseaux temporaires dont les périodes inondées sont semblables avec le témoin permanent calme (partie amont du Destel). Ce résultat a été confirmé par les affinités relativement élevées entre les phases lotiques et stagnantes des ruisseaux temporaires qui ont des périodes de mise en eau équivalentes. 
L'examen détaillé des peuplements globaux des ruisseaux temporaires (sauf celui de Péruy ) et ceux des ruisseaux permanents à cours rapide montre que certains groupes rhéophiles sont absents des ruisseaux temporaires: groupe des Trichoptères rhéophiles (Rhyacophila dorsalis, Rhyacophila vulgaris, Hydropsyche instabilis, Silo nigricornis, Synagapetus dubitans, Hyporhyacophila sp.), certains Éphéméroptères (Ecdyonurus sp., Heptagenia lateralis), certains Plécoptères (Perla marginata). De plus, certains groupes sont mieux représentés dans les ruisseaux temporaires étudiés que dans les ruisseaux permanents à cours rapide; ce sont des Coléoptères Hydrocanthares, des Hétéroptères, Odonates et Crustacés planctoniques et cela, quelle que soit la vitesse de la phase lotique des ruisseaux temporaires.

La présence de ces groupes lénitophiles est directement liée à la physiographie du lit des ruisseaux temporaires; en effet, lorsque la phase lotique est rapide, il y a toujours des zones calmes propices à l'installation de ce type de faune.

Si on tient compte, d'une part de l'absence des espèces rhéophiles, d'autre part de la présence d'espèces limnophiles et enfin du fait que des espèces présentes à l'état larvaire sont ubiquistes, il n'est pas paradoxal de constater que les ruisseaux temporaires, quelle que soit la vitesse du courant pendant la phase lotique, sont plus proches des ruisseaux permanents calmes que des ruisseaux permanents à cours rapide.

\section{CONCLUSIONS}

Les ruisseaux, permanents et temporaires, étudiés sont situés le long d'un axe NO-SE de $46 \mathrm{~km}$; ils sont soumis à des influenccs climatiques différentes : Roques Hautes et Subéroque sont les ruisseaux les plus septentrionaux, Destel est le plus méridional et Péruy occupe une position géographique intermédiaire.

$\mathrm{Si}$, du fait de leur turbulence, les deux ruisseaux permanents lotiques de référence (Roques Hautes et la partie amont de Péruy) ne sont que très peu tributaires des conditions climatiques (températures et débits assez constants), le ruisseau permanent lentique témoin est directement soumis aux variations saisonnières des températures mais son débit varie peu. On observe également une stabilité des propriétés chimique de l'eau, que ce soit dans l'espace (stations 1 et 2 du ruisseau de Roques Hautes) ou dans le temps (prélèvements annuels).

Par contre, les trois ruisseaux temporaires (Subéroque, partie aval de Péruy et du Destel) sont étroitement soumis aux conditions climatiques extérieures : les températures relevées dans ces 
ruisseaux varient avec celles de l'air atmosphérique et la période inondée est tributaire des précipitations et du niveau de la nappe phréatique. Ainsi, les précipitations d'automne font remonter le niveau de la nappe phréatique du ruisseau du Destel et la remise en eau se fait progressivement dans les dépressions avant l'installation d'un courant faible. La remise en eau est brutale dans le ruisseau de Subéroque et dans la partie aval du ruisseau de Péruy, elle correspond à un mouvement d'eau de l'amont vers l'aval avec l'installation d'un courant souvent rapide. Les deux ruisseaux temporaires (Subéroque et partie basse de Péruy) diffèrent cependant par la date de la remise en eau; elle se fait immédiatement après les pluies d'automne pour le premier, dans le courant de l'hiver pour le second. Comme les températures et le débit, les éléments chimiques varient quantitativement dès que le courant est devenu trop faible pour assurer un renouvellement de l'eau et que l'évaporation et les précipitations commencen! à agir de façon antagoniste.

A l'issue de cette étude, on a pu mettre en évidence d'une part l'influence prépondérante de la date et de la durée de la mise en eau ainsi que nous l'avons déjà développé et l'influence du mode de remise en eau des ruisseaux temporaires.

Le ruisseau dont la nappe phréatique détermine la remise en eau (partie temporaire du ruisseau du Destel) renferme des espèces qu'on ne retrouve pas dans les ruisseaux dont la remise en eau se fait directement par les eaux de pluie (ruisseaux de Subéroque et de Péruy). Ces espèces sont représentées par des Crustacés : Asellus sp., des Planaires : Dugesia tigrina, des Mollusques : Ancylus sp. et des insectes Coléoptères : Oulimnius rivularis. Au début de l'été, ces espèces s'enfoncent avec la nappe phréatique lorsque la texture du substrat le permet.

Des observations faites au cours des mois de juillet et août 1972 nous ont permis de retrouver quelques individus apparemment inactifs d'Ancylus sp. au niveau de la frange d'humectation de galets enchâssés dans la terre humide ainsi que dans la partie supérieure de la nappe. A la même période, les individus des autres espèces sont actifs dans la nappe. Au moment de la remontée de la nappe phréatique, ces quatre espèces sont les premières à repeupler le biotope avant même l'installation d'un périphyton. Ces espèces survivent donc à la période sèche de ce ruisseau temporaire.

Les coefficients d'affinité ont montré que les ruisseaux temporaires étudiés étaient différents entre eux et différents des ruisseaux 'permanents de référence. Tout le problème consiste à multiplier les stations dans l'espace afin de déterminer si les ruisseaux temporaires peuvent être classés en quelques « unités» différentes ou si 
on peut mettre en évidence un «gradient» de ruisseaux temporaires depuis les ruisseaux permanents jusqu'aux ruisseaux éphémères. Une étude détaillée des ruisseaux du Sud de la France permettra sans doute de répondre à cette question et de mieux définir la notion de ruisseau temporaire.

\section{TRAVAUX GITES}

Arell (D. A.). 1959. - Observations on mosquito population of an intermittent foothill stream in California. Ecology, 40 (2) : 186193.

Aubert (J.). 1963. - Les Plécoptères des cours d'eau temporaires de la péninsule ibérique. Bull. Soc. Entomol. Suisse, 35 (3-4) : 301-15.

Bigot (L.). 1972. - Contribution à l'étude biocénotique de la garrigue à Quercus coccifera I. Vie el Milieu, 23 (sous presse).

Champeau (A.). 1971. - Originalité du peuplement en Copépodes dans les eaux temporaires de basse Provence et de Corse. Ann. Univ. Provence, $45: 55-80$.

Champeau (A.). 1971. - Recherches sur l'adaptation de la vie latente des Copépodes Cycliopoïdes et Harpacticoïdes des eaux temporaires de basse Provence. Bull. Soc. Ecol., 2 (2) : 1-20.

Cancela Da Fonseca (J. P.). 1966. - L'outil statistique en biologie du Sol. Rev. Ecol. Biol. Sol, 3 (3) : 381-407.

Chifford (H. F.). 1966. - The ecology of Invertebrates in an intermittent stream. Invest. Indiana Lakes and Streams, 7 (2) : 58-98.

Eu.Liote (J. M.). 1971. -- Some methods for statistical analysis of sample of benthic invertebrates. Freshwater biological assoc., 25.

HARRison (A. D.). 1966. - Recolonisation of an Rhodesian Stream after drought. Archiv. Hydrobiol., 62 (3) : 405-421.

Hinton (H. E.). 1953. - Some adaptations of Insects to environments that are alternately dry and flooded with some notes on the habits of Stratiomyidae. Trans. Soc. Brit. Ent., 11 (10:209-227.

Hynes (H. B. N.). 1941. - The taxonomy and ecology of the nymphs of British Plecoptera, with notes on adults and eggs. Trans. $R$. ent. Soc. Lond., 91, pp. 459-557.

Hynes (H. B. N.). 1958. - The effect of drought on the fauna of a small mountain stream in Wales. Verh, internat. Ver. Limnol, $13: 826-833$.

Kamler (E.), Riedel (W.). 1960. - The effect of Drought on the Fauna Ephemeroptera, Plecoptera and Trichoptera of a mountain stream. Polsk. Arch. Hydrobiol., 8 : 87-89.

Kноо (S. G.). 1964. - Studies on biology of stoneflies Capnia bifrons (Newman) and notes on the diapause in the nymphs of this species. Gewäss. Abwäss., 34-35 : 23-30.

Kноо (S. G.). 1968. - Experimental studies on diapause in stoneflies. I Nymphs of Capnia bifrons (Newman). II Eggs of Diura bicaudata (L.). Proc. Roy. Entomol. Soc. London. Ser. A, Gen. Entomol., $43: 40-56$.

Lehmкunt, (H. P.) . 1971, - Stoneflies (Plecoptera: Nemouridae) from temporary lentic Habitats in Oregon. Amer. Midl. Naturalist USA, 85 (2) : 514-515.

Moon (H. P.). 1956. - Observations on a small portion of drying chalk stream. Proc. Zool. Soc. London, $126: 327-333$.

Stehr (W. C.', Branson (J. W.), 1938. An ecological study of an inter. mittent stream. Ecology, $19: 294-310$. 